Young Children's Ability to Describe Intermediate Clothing Placement Breanne Wylie ${ }^{1}$, Stacia N. Stolzenberg ${ }^{2}$, Kelly McWilliams $^{3}$, Angela D. Evans ${ }^{1}, \&$ Thomas D.$$
\text { Lyon }^{4}
$$ \\ Brock University ${ }^{1}$ \\ Arizona State University ${ }^{2}$ \\ John Jay College of Criminal Justice ${ }^{3}$ \\ University of Southern California Gould School of Law ${ }^{4}$
}

In press, Child Maltreatment

Authors Note: This research was supported by the Eunice Kennedy Shriver National Institute of Child Health and Human Development Grant HD087695 grant to Dr. Thomas D. Lyon and the Council for Research in the Social Sciences funding from Brock University to Dr. Angela D. Evans. Thank you to the Jack and Nora Walker Centre for Lifespan Research and to all the families who participated in the study. Portions of this data were presented at the 2019 annual meeting of the American Psychology-Law Society. A special thank you to Louise de Wit for sewing all the tiny clothing for the figures. 


\begin{abstract}
Children's ability to adequately describe clothing placement is essential to evaluating their allegations of sexual abuse. Intermediate clothing placement (partially removed clothing) may be difficult for young children to describe, requiring more detailed explanations to indicate the location of clothing (e.g., the clothes were pulled down to the knees). The current study investigated 172 3- to 6-year-olds' descriptions of clothing placement when responding to commonly used questions (yes/no, forced-choice, open-choice, where), as well as children's onoff response tendencies when describing intermediate placement (i.e.., labeling the clothing as fully on or off). Results revealed that where questions were superior in eliciting intermediate descriptions, even for the youngest children. Children sometimes exhibited tendencies to describe intermediate placements as "on" or "off," which varied by question-type and clothing placement. The implications of the findings for interviewing young children about sexual abuse are discussed.

Keywords: clothing placement; child sexual abuse; question type; response biases; intermediate descriptions
\end{abstract}




\section{Young Children's Ability to Describe Intermediate Clothing Placement}

During investigations of alleged child sexual abuse, children are often asked about clothing placement (Stolzenberg \& Lyon, 2017). For example, examining several hundred transcripts, Stolzenberg and Lyon (2017) found that $80 \%$ of trials and $65 \%$ of forensic interviews included questions about clothing placement. These questions are important as they can help to assess children's allegations of abuse. Children are asked about the placement of their clothing (and the clothing of the suspect) as a means of assessing their credibility and in determining the abusiveness and severity of the alleged touching. To date, there is limited research on bestpractices for asking children about clothing placement (Stolzenberg \& Lyon, 2017; Stolzenberg et al., 2017). Therefore, the current study sought to examine children's ability to describe clothing placement in responses to different question types (yes/no, forced-choice, open-choice, and where questions). Furthermore, we assessed whether children exhibited response tendencies when describing clothing that was partially removed (i.e., systematically understating or overstating the extent to which clothing was removed by responding that the clothing was on or off).

Two important factors to consider when assessing children's understanding of clothing placement are the location of the clothing and the format of the question. Clothing placement can be categorized as either simple, when clothing is fully on or off, or intermediate, when clothing is partially removed. Furthermore, intermediate placement can range from slightly removed (e.g., shirt is unbuttoned) to almost fully removed from the body (e.g., shirt is on the wrists). Stolzenberg and colleagues (2017) found that children were quite good at describing simple placement, though they demonstrated difficulties when describing intermediate placement. Intermediate clothing placement may be particularly difficult for children to describe, as the 
clothing is neither fully on nor off, and therefore requires more detailed explanations to describe the spatial location of the clothing.

In terms of question format, past research suggests that certain types of questions may lead to underinformative responses. For example, in the context of clothing placement, attorneys and interviewers most commonly ask yes/no (e.g., Was your shirt on?) and forced-choice questions (e.g., Was your shirt on or off?), leading children to exhibit formal reticence whereby children provide minimal details in response to the question (Stolzenberg \& Lyon, 2017). That is, children often provide unelaborated yes or no responses to yes/no questions (Stolzenberg \& Lyon, 2014), and simply choose one of the two options provided in a forced-choice question (Peterson \& Grant, 2001), even when a more detailed description better describes the placement (e.g,. such as when clothing is partially off and removed to a joint). As such, researchers have examined the usefulness of open-choice questions and wh-questions to improve the informativeness of children's reports. Open-choice questions include a third option of 'something else' (e.g., Was your shirt on or off or something else?), allowing children to go beyond simply selecting one of the two forced options (see Anderson et al., 2010). However, whquestions are most often recommended, such as asking where the clothing was placed, as whquestions are less suggestive and allow children to self-generate information (Lamb et al., 2018).

To examine the influence of both clothing location and question type on children's descriptions of clothing placement, Stolzenberg and colleagues (2017) presented 3- to 6-yearolds with figurines with clothing in simple and intermediate positions and asked them to describe the location of the clothing using one of four question types: yes/no, forced-choice, open-choice, and where questions. As noted above, children were quite good at describing simple clothing placement (article of clothing is fully on or off), as accuracy was above $80 \%$ across all question 
types. However, when asked about intermediate placement, children were much more likely to provide an intermediate response (as opposed to simply responding "on" or "off") when asked a where question (70\% intermediate responses) than when asked to yes/no, forced-choice, and open-choice questions (10\% intermediate responses).

When questioning young children about clothing placement, it is also important to consider developmental differences in children's abilities to understand and adequately answer the question. For example, although children may generally be successful in responding to where questions, the youngest children may experience the greatest difficulties with this question form. It is possible that preschool children may experience difficulties generating information to answer a where question due to deficient recall abilities. Furthermore, past research suggests that comprehension develops before production (e.g., Bates, 1993; Sansavini et al., 2010), suggesting that young children may understand, but not yet produce, the language required to describe clothing placement when answering a where question. Given these developmental differences, it is important to assess changes in children's ability to answer questions about clothing placement as children age. This information may offer insight into developmentally appropriate questioning practices.

Stolzenberg and colleagues (2017) could not fully assess age differences because many of the youngest children (3- to 4-year-olds) were excluded from analyses. In their task, children described visible figurines. As a result, $25 \%$ of the youngest children simply pointed, or said "right there," in response to where questions about clothes on the figurines, what the authors called deictic responding. Given that pointing demonstrated a lack of understanding for the task, as children were asked to describe the clothing placement to a research assistant (RA) who was behind a screen and could not see the figurine, consistent deictic responders were excluded from 
analyses. This resulted in a smaller sample size for the where condition (yes/no, $\mathrm{n}=23$; forcedchoice, $\mathrm{n}=24$; open-choice, $\mathrm{n}=26$; where, $\mathrm{n}=18$ ), and in turn may have inflated the apparent efficacy of where questions with younger children.

Deictic responding may reflect two aspects of immaturity in children's ability to complete Stolzenberg and colleagues' (2017) clothing task. First, deictic responses may indicate limited theory-of-mind skills, in failing to take on the RA's naive perspective and recognize that because the RA was behind the screen, pointing was uninformative. It is also possible that deictic responses indicate limited verbal abilities, as children may use pointing as a response strategy when they do not possess the language required to describe clothing placement. In this case, it is probable that children with limited verbal abilities would perform poorly when responding to where questions, as these questions require the child to generate their own response (unlike yes/no, forced-choice, and open-choice questions). If the latter explanation is true, and deictic responding is related to verbal proficiency, this could undermine the actual efficacy of where questions.

Another important problem to consider in children's responding is the potential for response tendencies, in which children consistently label clothing as on or off, depending on question type and clothing placement. Children might associate the term 'on' with clothing being in contact with the body, leading them to label partially removed clothing with a simple 'on' response. This in turn could lead to underestimating the extent to which clothing was removed. In contrast, children might focus on the displacement of the clothing, and label partially removed clothing as 'off,' thus exaggerating the removal of clothing.

These response tendencies can be particularly important in cases of alleged sexual abuse. If clothing placement was in fact intermediate, describing clothing as "on" suggests that abuse 
may not have occurred or may have been less severe (e.g., touching may not be abusive, penetration could not occur). On the other hand, describing clothing as "off" could lead nonabusive actions to sound abusive (e.g., toileting may sound suspicious if clothing was entirely removed). In addition, a tendency to describe intermediate placement as "off" could undermine the credibility of an abuse claim (e.g., stating clothing was fully off in a rushed, crowded, or public space is less plausible, and in turn may not be believed). In the study by Stolzenberg and colleagues (2017), children demonstrated some tendency to give "on” responses (e.g., when clothing was at the elbows or knees (mid-joint), 64\% of children demonstrated an on bias when answering yes/no questions). However, there was insufficient power to examine response tendencies across all question types. Therefore, this study will examine children's "on" and "off" response tendencies to intermediate clothing placement. These response tendencies differ from the standard concerns about response biases (e.g., always answering "yes," always answering "no," or always choosing the first or last choice when asked forced-choice questions).

\section{The Current Study}

The present study examined the influence of clothing placement (on, unfastened, midjoint: elbow/knee, low-joint: wrist/ankle, off), question type (yes/no, forced-choice, open-choice, where), and age on children's ability to adequately describe clothing placement. Children 3 to 6 years of age viewed figurines, experienced a five second delay, and then were asked questions about clothing placement. Because the figurines were no longer visible when children answered questions, deictic (pointing) responses were prevented. First, it was expected that children would find it easier to describe simple placement than intermediate placement. Additionally, since children often provide unelaborated responses to yes/no questions (Stolzenberg \& Lyon, 2014), and tend to simply choose one of the responses in forced-choice and open-choice questions 
(Peterson \& Grant, 2001), we predicted that children's performance when describing intermediate clothing placement would be superior when answering where questions compared to all other formats. We did not expect any differences between yes/no, forced-choice, and openchoice questions. Furthermore, given that children develop spatial language with age (Clark, 2016), we expected that older children would provide more adequate descriptions of clothing placement than younger children.

In terms of response tendencies, given that Stolzenberg and colleagues (2017) found some evidence of preference for describing intermediate placement as “on”, we tentatively predicted that children would exhibit an "on" response tendency when describing intermediate placement.

\section{Method}

\section{Participants}

One hundred and seventy-two 3- to 6-year-olds ( $M_{\text {age }}=4$ years, 11 months, $S D=14$ months, 50\% males) participated in the current study, including 863 - to 4-year-olds $\left(M_{a g e}=4\right.$ years, 0 months, $S D=7$ months, $52 \%$ males) and 865 - to 6 -year-olds $\left(M_{\text {age }}=6\right.$ years, 0 months, $S D=7$ months, $48 \%$ males). Twenty-seven additional participants were excluded for choosing not to complete the tasks ( $M_{a g e}=4$ years, 6 months, $57 \%$ males $)$; this was likely due to the clothing task being the last task during the testing session. Participants were recruited from the community through a database of families interested in participating in research. Total household income was collected as an indicator of economic status, with the majority of households (67\%) reported an income over $\$ 75,000$ (5\% did not report total household income). The ethnic background identified by participants in the sample included $84 \%$ Caucasian, $1 \%$ Asian, $1 \%$ South-Asian, 2\% African-Canadian, 1\% Hispanic, 10\% as other, and 1\% did not report child 
ethnicity. This study was approved by the University ethics board. Prior to beginning testing, written consent was obtained by all parents and verbal assent from all children.

\section{Materials and Procedure}

All children were tested individually in an on-campus research laboratory. Children first completed a battery of tasks not related to the current study. These tasks took approximately 30 minutes to complete. Next, children completed the Woodcock-Johnson IV test of oral language picture vocabulary task (WJ IV, Schrank, McGrew, \& Mather, 2014), to assess children's language proficiencies. Children were presented with a series of pictures and asked to label each picture. The task was terminated when six consecutive pictures were incorrectly labelled. Children received one point for each correctly identified picture. Scores can range from 0 to 54.

Finally, all children completed the clothing task using modified methodology from Stolzenberg and colleagues (2017), where they were shown 30 pictures of figurines on a laptop computer screen with shirts or pants in varying positions. The clothing task took approximately 10 minutes to complete. Each picture was presented for 5 seconds, followed by a blank computer screen. The 30 pictures of figurines included 6 items of clothing ( 3 shirts: blue, white, purple; 3 pants: blue, green, purple), shown in 5 different placements: 2 simple placement (on and off) and 3 intermediate placements (unfastened, with shirt totally open or fly open and spread apart; midjoint, with shirt at elbows or pants at knees; low-joint, with shirt at wrists or pants at ankles). Therefore, altogether children were shown 12 simple and 18 intermediate clothing placements. The order of presentation for shirts (15 pictures) and pants (15 pictures) were counterbalanced between participants. Within the pictures of shirts and pants, the order of presentation for clothing color and clothing position was randomized. After each picture of the figurine disappeared from the computer screen, children were asked to identify the location of the 
clothing in one of four randomly assigned, between-subjects, question type conditions: yes/no (e.g., Is the shirt/Are the pants on?, Is the shirt/Are the pants off?), forced-choice (e.g., Is the shirt/Are the pants on or off?), open-choice (e.g., Is the shirt/Are the pants on, or off, or something else?), and where (e.g., Where is the shirt/are the pants?). For yes/no, forced-choice, and open-choice questions, we controlled for response biases (e.g., yes-bias, no-bias, last-choice bias) by presenting children with both options (on and off) and counterbalancing the order of these terms within participants (with the exception of the 'something else' option, which was always presented last). Altogether the procedure took approximately 45 minutes.

\section{Coding}

Response type. Children's responses in the clothing task were coded as on, off, something else, intermediate, incomplete, or "I don't know." Intermediate responses included descriptions of clothing placement that could not be captured by a single spatial term, and included providing a preposition in conjunction with a body part (e.g., for shirt, "on his/her shoulders/arms/elbows/hands"; for pants, "on his/her legs/knees/feet"), or a description of clothing displacement (e.g., "unbuttoned”, “pulled down”). Incomplete responses were either inaccurate, insufficient, or off-topic descriptions.

Given that yes/no questions were unique in that children were asked two questions, responses were coded as on or off when children affirmed one, but not the other single spatial term: on (e.g., "Is the shirt on?" "Yes", "Is the shirt off?" "No"), or off (e.g., "Is the shirt on?" "No", "Is the shirt off?" "Yes"). When children affirmed or denied both terms, children's responses were coded as a double yes (e.g., "Is the shirt on?" "Yes", "Is the shirt off?" "Yes"), or double no response (e.g., "Is the shirt on?" "No", "Is the shirt off?" "No"). If children provided 
an intermediate response to one of the two yes/no questions, their response was coded as intermediate (e.g., "Is the shirt on?" "It is on his elbows," "Is the shirt off?" "No").

Adequacy. For the coded responses, inter-rater reliability was assessed for $100 \%$ of the sample (Kappa > .90). The adequacy of children's responses was then assessed. For simple clothing placement, children received a score of 0 to 12 for the number of trials correctly labelled as simply on or off. For intermediate clothing placement, children received a score of 0 to 18 for the number of trials labelled with an appropriate intermediate response. All other responses for intermediate clothing placement were scored as inadequate because they failed to convey the fact that the clothes were neither entirely on nor off.

\section{Results}

\section{Preliminary}

Preliminary analyses revealed that for simple placement, the proportion of children's adequate responses were not significantly different for shirts $(M=.89, S D=.21)$ and pants $(M=$ $.88, S D=.21), t(171)=.82, p=.415$, CI $95 \%[-.02, .04]$. Similarly, for intermediate placement, the proportion of children's adequate responses were not significantly different for shirts $(M=$ $.18, S D=.31)$ and pants $(M=.19, S D=.30), t(171)=.91, p=.365$, CI $95 \%$ [-.05, .02]. Therefore, all further analyses collapsed across shirts and pants. Preliminary analyses also revealed that, controlling for age, children's verbal ability (WJ scores) was not significantly related to adequacy in describing simple or intermediate clothing placement across all question types, with the exception of simple placement for open-choice questions $(\mathrm{r}=.436, p=.004)$.

Next, we descriptively examined children's response patterns to assess how children were responding across all question types and clothing placements (see Table 1). Depicting all possible response options offers an overall understanding of response patterns. 


\section{Adequacy}

First, we assessed the adequacy of children's responses in describing clothing placement, which consisted of simple "on" and "off” responses to questions about simple placement, and intermediate responses to questions about intermediate placement. We examined the effects of question type, clothing position, and age on the proportion of children's adequate responses, using a 4 (Question Condition: Yes/No, Forced-Choice, Open-Choice, Where) by 2 (Placement: Simple, Intermediate) by 2 (Age: Younger, Older) repeated measures ANCOVA, with proportion of adequate responses as the dependent variable, and Woodcock Johnson Scores entered as a covariate. There was no significant main effect of age, $F(1,163)=1.30, p=.255, \eta_{\mathrm{p}}^{2}=.01$, and no significant interactions with age, $p \mathrm{~s}>.05$. There was a significant main effect of question condition, $F(3,163)=19.20, p<.001, \eta_{\mathrm{p}}{ }^{2}=.26$, and a significant main effect of placement, $F(1,163)=31.44, p<.001, \eta_{\mathrm{p}}^{2}=.16$. These main effects were qualified by a significant question condition by placement interaction, $F(3,163)=36.60, p<.001, \eta_{\mathrm{p}}{ }^{2}=.40$. Simple effects tests were used to investigate the influence of question type on children's adequacy, separately for simple and intermediate clothing placement. For simple clothing placement, there were no significant differences in children's adequacy when answering yes/no, forced-choice, openchoice, and where questions $(M=.89, S D=.37 ; M=.94, S D=.37 ; M=.89, S D=.37 ; M=.86$, $S D=.37$, respectively), $p \mathrm{~s}>.05$. For intermediate clothing placement, children were significantly more likely to provide an adequate response when answering a where question $(M=$ $.51, S D=.39)$, compared to a yes/no, forced-choice, and open-choice questions $(M=.07, S D=$ $.39 ; M=.07, S D=.39 ; M=.11, S D=.39$, respectively), $p s<.001$ (see Intermediate column in Table 2). There were no significant differences between yes/no, forced-choice, and open-choice questions, $p \mathrm{~s}>.05$. 


\section{Response Tendencies}

Next, when children failed to provide an intermediate response to intermediate placements (see Simple and Other Column in Table 2), we were interested in whether children exhibited a tendency to describe intermediate clothing placement using simple placement terms (on response tendency or off response tendency). We created proportion scores for children's responses (the total number of "on" responses, divided by the sum of "on" and "off" responses), for which above $50 \%$ represents an on response tendency and below $50 \%$ represents an off response tendency. T-tests were calculated for the proportion scores for each question type and intermediate position, with the test value of .50 (see Table 3 for mean proportion scores, standard deviations, and inferential data). For all question types, when clothing was unfastened children exhibited an on response tendency. For yes/no, forced-choice, and open-choice questions, when clothing was at the mid-joint (elbow, knee) and the low-joint (wrist, ankle) children exhibited an off response tendency. For where questions, children exhibited an on response tendency when clothing was at the mid-joint, and no significant tendency when clothing was at the low-joint.

\section{Exploratory analyses}

We conducted exploratory analyses to better understand two unexpected findings. Given the surprising lack of age differences, we further examined younger children's response patterns. We had expected that prior research (Stolzenberg et al., 2017) may have exaggerated young children's performance, given that many younger children were excluded from analyses due to deictic responding. However, results from our study reveal that younger children's response patterns are quite similar to the general pattern of children's responses (see Table 4 for younger children's response pattern, in comparison to Table 1 collapsed across age groups). For example, where questions still appear superior across question types. 
Second, we conducted an exploratory analysis to better understand children's response tendencies. At the same time that where questions elicited more intermediate responses than other question types, when children failed to give an intermediate response, they were more likely to characterize the clothes as "on" than "off." We suspected that children's "on" responses to where questions might reflect elliptical responses; that is, that they might be responding "on" because an adequate intermediate response would likely start with the word "on" (e.g., "on his knees" or "on his arms"). We created proportion scores for the number of times children began an intermediate response with the word "on" or "off." Consistent with our speculation, across all question types children's intermediate responses were significantly more likely to include the term "on" $(M=.40, S D=.41)$ than the term "off" $(M=.11, S D=.27), t(84)=4.97, p<.001$.

\section{Discussion}

The purpose of the present study was to assess how young children respond to questions about clothing placement, comparing where questions to yes/no,forced-choice, and open-choice questions. Overall, children were quite good at describing simple clothing placement (clothing that is fully on or off the body), though they experienced difficulties when describing intermediate clothing placement (partially removed). However, where questions were superior in eliciting intermediate descriptions compared to yes/no, forced-choice, and open-choice questions, even among the youngest children. Furthermore, when children failed to provide intermediate descriptions, they exhibited a tendency to describe clothing as "off" when answering yes/no, forced-choice, and open-choice questions about clothing at the mid- and low-joint, and a tendency to describe clothing as "on" when answering where questions about clothing at the mid-joint. In what follows, we elaborate on the findings, and then discuss the limitations, future directions, and implications for practice. 


\section{Support for the Superiority of Where Questions}

First, we examined children's ability to adequately describe simple and intermediate clothing placement as a function of question type. When clothing was simply placed (e.g., fully on or fully off the body), children did quite well, regardless of question form. Rates of adequate responding were quite high, ranging from $83 \%$ (open-choice condition when the clothing was fully off) to $95 \%$ (forced-choice condition when the clothing was fully off). Yet, when clothing was intermediately placed, the form of the question did matter; children in the where condition were significantly more likely to provide an adequate description than children in any other question condition. The difference was large, with children providing adequate responses to the where questions $51 \%$ of the time, compared to 7 to $11 \%$ in response to the other question types.

The findings are consistent with Stolzenberg and colleagues (2017), who similarly found that where questions were superior in eliciting intermediate descriptions. However, in that study, many of the youngest children answering where questions about clothing placement had to be excluded because they gave deictic (pointing) responses. This study imposed a delay in order to prevent deictic responding, and where questions continued to elicit better responding.

Remarkably, we did not find age differences; adequate response rates did not differ for 3to 6-year-olds. Furthermore, when we looked more closely at 3- to 4-year-olds responses, they looked similar to the general pattern of children's responses, suggesting that where questions were superior even among the youngest children.

\section{Response Tendencies}

When children did experience difficulties in describing intermediate clothing placement, we were interested in whether children would exhibit tendencies to answer either "on" or "off." That is, we examined whether children would systematically appear to understate or exaggerate 
the extent to which clothing was removed. In cases of alleged sexual abuse, response tendencies would be problematic for both "on" responses (e.g., saying the clothes were on suggests that abuse may be less severe or could not have occurred) and "off" responses (e.g., exaggerating the extent that clothing was removed may suggest non-abusive actions are abusive). Children in all question conditions exhibited a tendency to respond "on" when the clothing was unfastened (e.g., an open shirt fully unbuttoned or open pants fully unzipped); the percentage of "on" responses ranged from $72 \%$ to $100 \%$.

As the clothing moved off the body, towards the mid-joint (elbows or knees) or low-joint (wrists or ankles), children's on tendency disappeared, turning into a tendency to say "off." This was true for all question conditions except for children in the where condition, who exhibited some tendency to give "on" responses when they failed to give intermediate responses to midjoint placement. We suspected that children who responded "on" may have been giving elliptical answers (e.g., responding "on" when they meant "on the knees"). Consistent with this explanation, we found that children were more likely to use "on" (40\%) when successfully describing intermediate placement than “off” (11\%). Despite this tendency, children's overall rate of "on" responses to mid-joint placement was not higher for where questions (20\%) than other question types (e.g., yes/no elicited 25\%). That is, their greater tendency to say "on" than "off" was due to the rarity with which they said "off." Nevertheless, the finding highlights the need to treat young children's responses to where questions with some caution.

\section{Open-Choice Questions}

In response to open-choice questions, children provided more "something else" responses when describing intermediate placement (40\%) than when describing simple placement (9\%), which suggests that open-choice questions have some value in helping children signal when 
clothes are neither totally on nor off. Prior research has found that when neither choice in a forced-choice question is correct, including the "something else" option can more than double the accuracy of children's responses, though error rates remain high (London, Hall, \& Lytle, 2017).

Nevertheless, the value of open-choice questions is limited by the fact that the interviewer must follow-up a child's reference to "something else." It is likely that the interviewer would need to ask a where question, and that question could have been asked to begin with. Future work is needed to examine the utility of open-choice questions, including a direct comparison of simply asking where questions to asking open-choice questions with follow-ups.

We found that children with limited vocabulary performed poorly on open-choice questions about simple placement. This suggests that they exhibited a tendency to choose the last proffered choice, since the "something else" option was always given last. Research has found that young children exhibit a last-response bias when asked forced-choice questions (Mehrani \& Peterson, 2015). In this study, we controlled for last-response bias in response to the forcedchoice questions by alternating the last choice, but we always provided the "something else" option last in the open-choice questions because placing it earlier would seem unnatural. This problem suggests a disadvantage to open-choice questions with younger children.

\section{Limitations and Future Directions}

Although where questions more often elicited adequate descriptions of intermediate clothing placement (51\% were intermediate responses) compared to other question types (less than $11 \%$ were intermediate responses), intermediate placement remained difficult for young children to describe. Future work can determine whether young children may provide better 
descriptions in response to other types of questions (such as "what happened to his/your clothes") or whether they can be encouraged to elaborate on their sometimes elliptical responses to where questions.

It is possible that this study misestimated young children's ability to describe intermediate placement when it occurs in the context of potentially abusive experiences. Obviously, children questioned about potential abuse will be asked about clothing placement after a much longer delay than the delays they experienced in this study, and forgetting could change the relative advantages of where and other question types. In actual investigations children will be questioned about clothing placement on their own bodies (and on the bodies of suspects), and their ability to do so may be quite different than their ability to describe clothing placement on dolls. On the one hand, when potentially abusive actions occur, children may have limited visual access to clothing placement, particularly their own, but on the other hand, children may feel and hear clothing being displaced and this may facilitate their descriptive ability. Furthermore, children may be better able to describe their own clothing placement compared to others, as past research suggests participating in an event (as compared to observing an event) improves memory (Baker-Ward et al., 1990; Goodman \& Tobey, 1992; Murachver et al., 1996). Stress at the time of encoding may also affect children's subsequent abilities to provide complete descriptions, and stress may have both facilitative and debilitating effects (Chae, et al., 2018).

A conclusion that where questions are clearly superior must therefore be tempered by the limited ecological validity of the current study, and future work can examine children's ability to describe clothing placements after longer delays and in more interactive contexts. However, the current findings are consistent with an examination of children's answers to clothing placement 
questions in forensic interviews and criminal trials: children gave more intermediate responses when asked a wh- question (28\%) than when asked a yes/no $(3 \%)$ or forced-choice $(6 \%)$ question about clothing placement (Stolzenberg \& Lyon, 2017). In those cases, ground truth could not be determined (that is, whether clothing placement was in fact intermediate), and children were not randomly assigned to question-types, making the apparent superiority of $w h$ - questions equivocal. Taken in combination, this study, prior laboratory work (Solzenberg et al., 2017), and observational work (Stolzenberg \& Lyon, 2017) provides clear support for the superiority of where questions.

\section{Conclusion}

These findings have important implications for practitioners and legal professionals who interview young children about sexual abuse. While yes/no, forced-choice, and open-choice questions often lead young children to erroneously describe intermediate clothing placement as “on" or "off," questions asking where clothes were placed are more likely to elicit descriptions that adequately convey the fact that clothes were neither completely on nor completely off. At the same time, even where questions are less than perfect, and children need encouragement to elaborate on brief responses about clothing placement. 


\section{References}

Anderson, J., Ellefson, J., Lashley, J., Miller, A. L., Olinger, A., Russell, A., Stauffer, J, \& Weigman, J. (2010). The cornerhouse forensic interview protocol: RATAC. Thomas $M$. Cooley Journal of Practical and Clinical Law, 12, 193-331.

Baker-Ward, L., Hess, T. M., \& Flannagan, D. A. (1990). The effects of involvement on children's memory for events. Cognitive Development, 5, 55-69.

Bates, E. (1993). Comprehension and production in early language development: Comments on Savage-Rumbaugh et al. Monographs of the Society for Research on Child Development, $58,222-242$.

Chae, Y., Goodman, M., Goodman, G. S., Troxel, N., McWilliams, K., Thompson, R. A., ... \& Widaman, K. F. (2018). How children remember the Strange Situation: The role of attachment. Journal of Experimental Child Psychology, 166, 360-379.

Clark, E. V., (2016). First language acquisition (3rd ed.). Cambridge University Press.

Lamb, M. E., Brown, D. A., Hershkowitz, I., Orbach, Y., \& Esplin, P. W. (2018). Tell me what happened: Questioning children about abuse (2nd ed.). Wiley-Blackwell.

London, K., Hall, A. K. \& Lytle, N. E. (2017). Does it help, hurt, or something else? The effect of a something else response alternative on children's performance on forced-choice questions. Psychology, Public Policy, \& Law, 23, 281-289. http://dx.doi.org/10.1037/law0000129

Mehrani, M., \& Peterson, C. (2015). Recency tendency: Responses to forced-choice questions. Applied Cognitive Psychology, 29, 418-424. 
Murachver, T., Pipe, M. E., Gordon, R., Owens, J. L., \& Fivush, R. (1996). Do, show, and tell: Children's event memories acquired through direct experience, observation, and stories. Child Development, 67, 3029-3044.

Peterson, C. \& Grant, M. (2001). Forced-choice: Are forensic interviewers asking the right questions? Canadian Journal of Behavioural Science, 33, 118-127.

Sansavini, A., Bello, A., Guarini, A., Savini, S., Stefanini, S., \& Caselli, M. C. (2010). Early development of gestures, actions, word comprehension and word production and their relationship in Italian infants: A longitudinal study. Gesture, 10, 52-58.

Schrank, F. A., McGrew, K. S., \& Mather, N. (2014). Woodcock-Johnson IV Tests of Cognitive Abilities. Rolling Meadows, IL: Riverside.

Stolzenberg S. N., \& Lyon, T. D. (2014). How attorneys question children about the dynamics of sexual abuse disclosure in criminal trials. Psychology, Public Policy, \& Law, 20, 19-30.

Stolzenberg, S. N., Lyon, T. D. (2017). 'Where were your clothes?' Eliciting descriptions of clothing placement from children alleging sexual abuse in criminal trials and forensic interviews. Legal and Criminological Psychology, 22, 197-212. https://doi.org/10.1111/lcrp.12094.

Stolzenberg, S. N., McWilliams, K., Lyon, T. D. (2017). Spatial language, question type, and young children's ability to describe clothing: Legal and developmental implications. Law and Human Behavior, 41, 398-409. https://doi.org/10.1037/lhb0000237.

Tobey, A. E., \& Goodman, G. S. (1992). Children's eyewitness memory: Effects of participation and forensic context. Child Abuse \& Neglect, 16, 779-796. 
Table 1.

Proportion of Responses as a Function of Condition and Clothing Placement

\begin{tabular}{|c|c|c|c|c|c|c|c|c|c|}
\hline 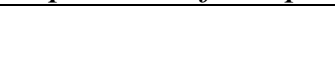 & \multicolumn{9}{|c|}{ Responses } \\
\hline & On & Off & $\begin{array}{l}\text { Something } \\
\text { else }\end{array}$ & $\begin{array}{c}\text { Double } \\
\text { Yes }\end{array}$ & $\begin{array}{c}\text { Double } \\
\text { No }\end{array}$ & Intermediate I & Incomplete & IDK & $\begin{array}{l}\text { Non- } \\
\text { responsive }\end{array}$ \\
\hline \multicolumn{10}{|l|}{ Yes/No } \\
\hline On & .87 & .04 & - & .07 & .01 & - & .004 & - & .004 \\
\hline Unfastened & .58 & .20 & - & .09 & .05 & .06 & .02 & - & - \\
\hline Mid-joint & .25 & .53 & - & .09 & .03 & .10 & .02 & - & - \\
\hline Low-joint & .19 & .62 & - & .10 & .03 & .04 & .02 & - & - \\
\hline Off & .04 & .88 & - & .03 & .05 & - & .004 & - & - \\
\hline \multicolumn{10}{|l|}{ Forced-Choice } \\
\hline On & .93 & .07 & - & - & - & - & - & .004 & - \\
\hline Unfastened & .61 & .29 & - & - & - & .07 & .02 & .01 & .004 \\
\hline Mid-joint & .25 & .67 & - & - & - & .06 & .02 & .004 & .004 \\
\hline Low-joint & .22 & .70 & - & - & - & .07 & .004 & - & .01 \\
\hline Off & .04 & .95 & - & - & - & - & .01 & .004 & - \\
\hline \multicolumn{10}{|l|}{ Open-Choice } \\
\hline On & .88 & .05 & .05 & - & - & - & .01 & - & .01 \\
\hline Unfastened & .38 & .15 & .37 & - & - & .08 & .01 & - & .01 \\
\hline Mid-joint & .05 & .34 & .44 & - & - & .14 & .02 & .01 & .004 \\
\hline Low-joint & .05 & .47 & .36 & - & - & .10 & .02 & .004 & .004 \\
\hline Off & .03 & .83 & .13 & - & - & - & .01 & - & .004 \\
\hline \multicolumn{10}{|l|}{ Where } \\
\hline On & .88 & - & - & - & - & - & .10 & .01 & .01 \\
\hline Unfastened & .41 & - & - & - & - & .32 & .22 & .04 & .01 \\
\hline Mid-joint & .20 & .03 & - & - & - & .60 & .15 & .01 & .01 \\
\hline Low-joint & .10 & .06 & - & - & - & .65 & .16 & .02 & .01 \\
\hline Off & .03 & .86 & - & - & - & - & .08 & .01 & .02 \\
\hline
\end{tabular}

Note. (-) represents no response 
Table 2.

Proportion of Simple, Intermediate, and Other Responses to Intermediate Clothing Placement by Question Type.

\begin{tabular}{llll} 
& Simple & Intermediate & Other \\
\hline Yes/No & .79 & .07 & .15 \\
Forced Choice & .91 & .07 & .02 \\
Open Choice & .47 & .11 & .42 \\
Where & .28 & .51 & .21 \\
\hline Note. 'Other' responses include 'something else', incomplete', 'IDK', and 'non-responsive'
\end{tabular}

Note. 'Other' responses include 'something else', incomplete', 'IDK', and 'non-responsive' responses.

Table 3.

Response Bias as a Function of Condition and Clothing Placement

\begin{tabular}{ccccccc}
\hline & $M$ & $S D$ & $t$ & $d f$ & $p$ & $d$ \\
\hline Yes/No & & & & & & \\
Unfastened & .75 & .26 & 6.35 & 42 & $<.001$ & .97 \\
Mid-joint & .33 & .37 & 3.03 & 41 & .004 & -.47 \\
Low-joint & .24 & .33 & 5.10 & 42 & $<.001$ & -.78 \\
Forced-Choice & & & & & & \\
Unfastened & .70 & .33 & 3.92 & 40 & $<.001$ & .61 \\
Mid-joint & .26 & .34 & 4.62 & 42 & $<.001$ & -.71 \\
Low-joint & .24 & .36 & 4.63 & 41 & $<.001$ & -.72 \\
Open-Choice & & & & & & \\
Unfastened & .72 & .36 & 3.71 & 36 & .001 & .61 \\
Mid-joint & .15 & .29 & 6.38 & 27 & $<.001$ & -1.21 \\
Low-joint & .16 & .33 & 6.19 & 36 & $<.001$ & -1.02 \\
Where $\quad$ & & & & & \\
Unfastened & 1.00 & .00 & $*$ & $*$ & $*$ & $*$ \\
Mid-joint & .92 & .25 & 7.98 & 22 & $<.001$ & 1.66 \\
Low-joint & .69 & .45 & 1.63 & 14 & .126 & .42 \\
\hline
\end{tabular}

Note. Proportion scores represent the number of on responses, out of on and off responses, where above $50 \%$ reflects an on bias and below $50 \%$ reflects an off bias.

*Given that $S D=0$, t-test can not be calculated. 
Table 4.

Proportion of Responses as a Function of Condition and Clothing Placement, for Younger Children ( 3 and 4 years) Responses

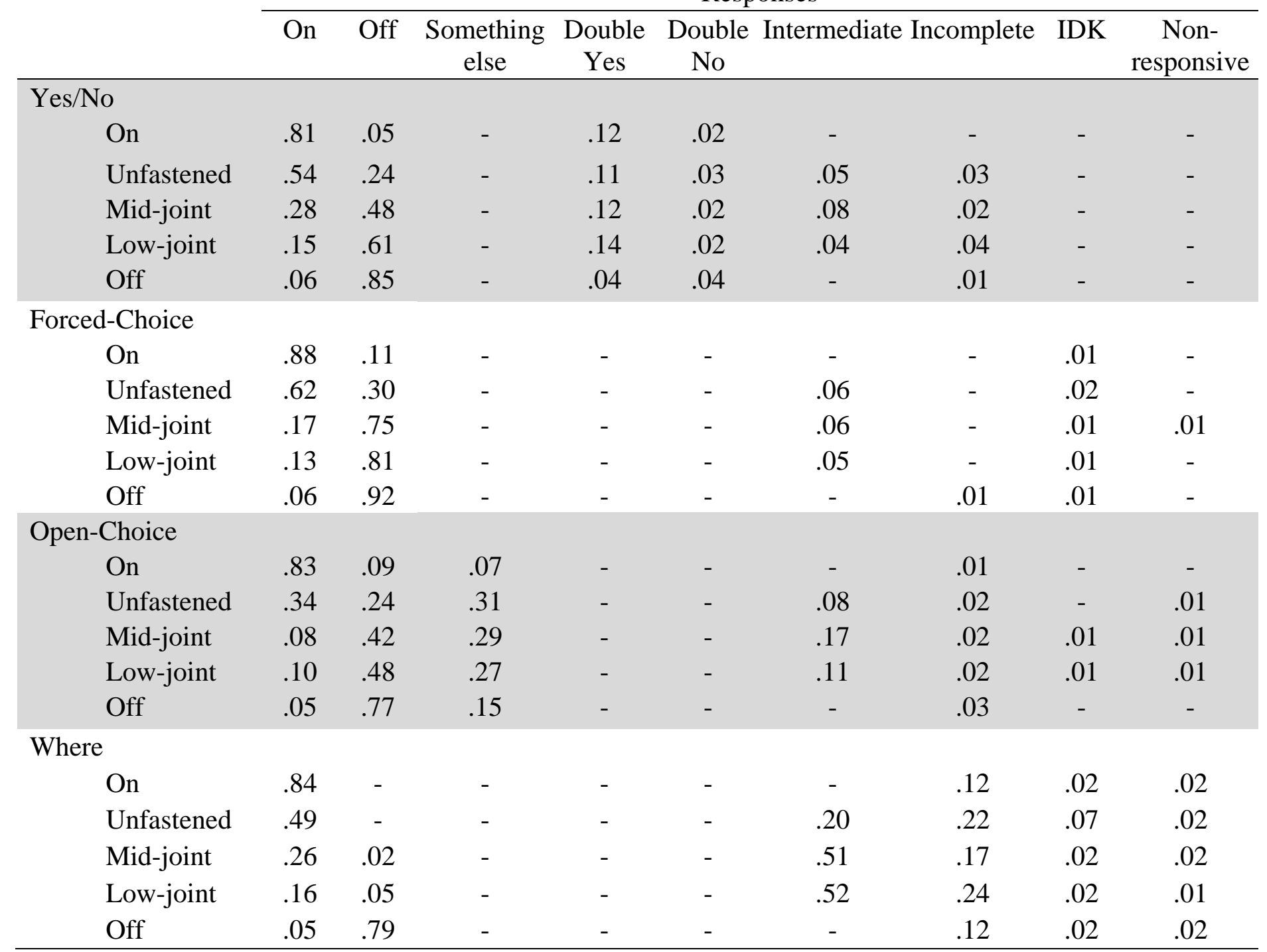

Note. (-) represents no response 